\title{
Giant electron-phonon interactions in molecular crystals and the importance of non-quadratic coupling
}

\author{
Bartomeu Monserrat,* Edgar A. Engel, and Richard J. Needs \\ TCM Group, Cavendish Laboratory, University of Cambridge, \\ J. J. Thomson Avenue, Cambridge CB3 OHE, United Kingdom
}

(Dated: September 29, 2015)

\begin{abstract}
We investigate electron-phonon coupling in the molecular crystals $\mathrm{CH}_{4}, \mathrm{NH}_{3}, \mathrm{H}_{2} \mathrm{O}$, and $\mathrm{HF}$, using first-principles quantum mechanical calculations. We find vibrational corrections to the electronic band gaps at zero temperature of $-1.97 \mathrm{eV},-1.01 \mathrm{eV},-1.52 \mathrm{eV}$, and $-1.62 \mathrm{eV}$, respectively, which are comparable in magnitude to those from electron-electron correlation effects. Microscopically, the strong electron-phonon coupling arises in roughly equal measure from the almost dispersionless high-frequency molecular modes and from the lower frequency lattice modes. We also highlight the limitations of the widely used Allen-Heine-Cardona theory, which gives significant discrepancies compared to our more accurate treatment.

PACS numbers: $63.20 . \mathrm{kd}, 71.15 . \mathrm{Mb}$
\end{abstract}

Electronic band gaps are crucial for studying the optical and electrical properties of semiconductors, and are therefore central to modern technologies such as photovoltaics and thermoelectrics. Density functional theory (DFT) [1-3] using local and semi-local density functionals [4-6] notoriously suffers from the band gap problem caused by the inability of standard local and semilocal exchange-correlation functionals to reproduce the derivative discontinuity with respect to particle number exhibited by the exact functional $[7,8]$. This typically leads to underestimation of band gaps. Computationally intensive techniques such as diffusion Monte Carlo (DMC) [9-11], GW many-body perturbation theory $[12,13]$, and hybrid exchange-correlation functionals [14-18] have been shown to greatly improve upon the accuracy of DFT band gaps, with corrections of several $\mathrm{eV}$ being common in some materials. However, even these more accurate methods assume a static lattice and neglect the effects of atomic vibrations.

One of the most striking consequence of electronphonon coupling in semiconductors is the temperature dependence of electronic band gaps [19]. Early experimental [20-23] and theoretical [24-26] efforts developed understanding of the temperature dependence of band gaps in standard semiconductors such as diamond, silicon and germanium. Recent interest has focused on first-principles calculations [27-37], which have mainly been used to study tetrahedral semiconductors, and particularly diamond due to the large electron-phonon coupling strength it exhibits. There is also great interest in electron-phonon coupling in nanocrystals [28, 33, 34], topological insulators [38, 39], and polymers [40].

Systematic studies of electron-phonon coupling over a wide range of classes of materials are currently lacking. Such studies could elucidate the interplay between the various microscopic properties that play central roles in electron-phonon coupling in semiconductors, and ultimately allow us to build an understanding that could be used to design new materials with tailored electronic and optical properties.

We gauge the strength of electron-phonon coupling by calculating the zero-point (ZP) vibrational correction to the electronic band gap. As far as we are aware, this is the first report of ZP corrections to the band gaps of the molecular crystals studied here. We find that the commonly used Allen-Heine-Cardona (AHC) theory is insufficient for studying electron-phonon coupling in the molecular crystals considered, and therefore we include terms beyond the AHC theory using a Monte Carlo sampling approach $[33,41]$. We find $\mathrm{ZP}$ corrections ranging from $-1.0 \mathrm{eV}$ in $\mathrm{NH}_{3}$, to $-2.0 \mathrm{eV}$ in $\mathrm{CH}_{4}$. These corrections are the largest observed to date, apart from those in hydrogen and helium at extreme pressures [41, 42]. The large ZP corrections arising from electron-phonon coupling demonstrate that studies of the electronic structure of semiconductors should include the effects of atomic vibrations even at zero temperature.

The ZP corrected band gap $E_{\mathrm{g}}$ may be written as the expectation value

$$
E_{\mathrm{g}}=\left\langle\Phi(\mathbf{q})\left|E_{\mathrm{g}}(\mathbf{q})\right| \Phi(\mathbf{q})\right\rangle,
$$

where $E_{\mathrm{g}}(\mathbf{q})$ is the value of the gap with atomic positions q (a $3 N$-dimensional vector in the vibrational phase space, which we describe in a basis of harmonic normal mode coordinates), and $|\Phi\rangle$ is the Gaussian vibrational ground state wave function. Equation (1) is based on adiabatic decoupling of the electronic and nuclear degrees of freedom, which has recently been explored by Patrick and Giustino [43], and shown to provide a semiclassical approximation to the ZP correction of band gaps.

In this work we evaluate the band gap correction of Eq. (1) using two different methods: the quadratic approximation and Monte Carlo sampling. In the quadratic approximation, the electronic band gap energy $E_{\mathrm{g}}$ is expanded about the equilibrium position in terms of har- 
monic vibrational mode amplitudes $q_{n \mathbf{k}}$, where $\mathbf{k}$ is the vibrational Brillouin zone wavevector and $n$ is the branch index. We work exclusively within the harmonic vibrational approximation, so that the wave function is symmetric and odd terms vanish in the expectation value:

$$
E_{\mathrm{g}}=E_{\mathrm{g}}(\mathbf{0})+\sum_{n, \mathbf{k}} a_{n \mathbf{k}}^{(2)}\left\langle\Phi_{n \mathbf{k}}\left|q_{n \mathbf{k}}^{2}\right| \Phi_{n \mathbf{k}}\right\rangle+\mathcal{O}\left(q^{4}\right)
$$

where $a_{n \mathbf{k}}^{(2)}$ are the diagonal quadratic expansion coefficients. Eq. (2) is equivalent to the AHC theory including off-diagonal Debye-Waller terms [44].

We gauge the strength of the electron-phonon coupling by calculating the $\mathrm{ZP}$ correction to the band gap. We calculate the couplings $a_{n \mathbf{k}}^{(2)}$ using a frozen phonon method, displacing the atoms by $\Delta q_{n \mathbf{k}}$ along harmonic vibrational modes in the positive and negative directions, and setting $a_{n \mathbf{k}}^{(2)}=\left(E_{\mathrm{g}}\left(\Delta q_{n \mathbf{k}}\right)+E_{\mathrm{g}}\left(-\Delta q_{n \mathbf{k}}\right)\right) / 2 \Delta q_{n \mathbf{k}}^{2}$. We have found that $a_{n \mathbf{k}}^{(2)}$ is sensitive to the vibrational amplitude used $\left(\Delta q_{n \mathbf{k}}\right)$, as shown in Fig. 1 and discussed below. This sensitivity is a signature of a strongly non-quadratic dependence of $E_{\mathrm{g}}$ on the normal mode amplitude $q_{n \mathbf{k}}$.

We also evaluate Eq. (1) using Monte Carlo sampling, with atomic configurations randomly drawn from the vibrational density. A Monte Carlo approach has an associated statistical uncertainty that can be reduced by using a large number of sampling points. The Monte Carlo approach allows us to include all higher-order terms neglected in Eq. (2). Here we use both approaches, and assess the validity of the quadratic approximation by comparing the results of the two methods. This comparison highlights the importance of higher-order terms and the inadequacy of the quadratic expansion for the molecular crystals considered. The only other known example of the failure of AHC theory has arisen in helium at terapascal pressures [41], and our work emphasizes that AHC theory can also be inadequate at ambient pressure. It is still valuable to use both approaches, as the quadratic expansion provides direct access to the microscopic physics, and therefore allows us to build a physical understanding of the origin of the strong electron-phonon coupling in these materials. Finally, we note that Brooks' theorem [45] breaks down if non-quadratic terms are important.

All our calculations have been performed using DFT and the pseudopotential plane-wave method as implemented in the CASTEP package [46]. We have used ultrasoft "on the fly" pseudopotentials to describe the electron-ion interaction [47]. We use an energy cutoff of $800 \mathrm{eV}$ and a Monkhorst-Pack [48] Brillouin zone sampling of spacing $2 \pi \times 0.03 \AA^{-1}$, that together lead to convergence of the difference between frozen-phonon structures to better than $10^{-4} \mathrm{eV}$ for the total energy per atom, $10^{-3} \mathrm{eV} / \AA$ for the forces on all atoms, $10^{-2} \mathrm{GPa}$
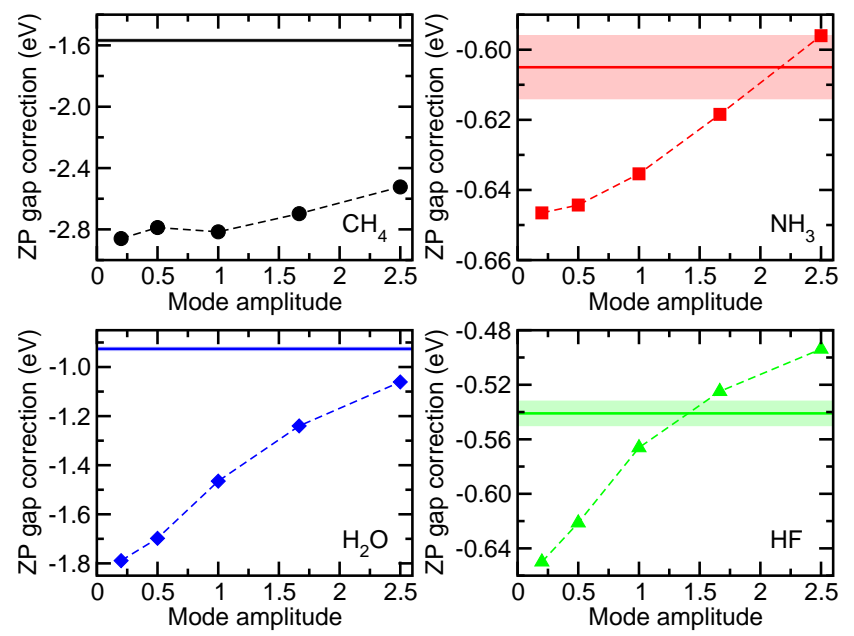

FIG. 1. (color online) ZP correction to the electronic band gaps for the molecular crystals $\mathrm{CH}_{4}, \mathrm{NH}_{3}, \mathrm{H}_{2} \mathrm{O}$, and $\mathrm{HF}$. The solid horizontal lines correspond to accurate Monte Carlo sampling, and the light bands represent their statistical uncertainty. The symbols correspond to results within the quadratic approximation, and the "Mode amplitude" refers to the amplitude at which the frozen phonon calculations have been performed. The mode amplitudes have units of $\sqrt{\left\langle q^{2}\right\rangle}=1 / \sqrt{2 \omega}$.

for the components of the stress tensor, and $10^{-4} \mathrm{eV}$ for the band gap. Unless otherwise stated, we report results using the PBE functional corrected with the TS scheme which describes dispersion interactions [49], as equilibrium volumes using this functional agree best with experimental volumes. Results obtained with the PBE [6] functional and the G06 [50] dispersion corrected functional are detailed in the Supplementary Material [51]. The ZP correction arising from electron-phonon coupling has some dependence on the functional and geometry used, but the central idea presented in this paper - the large strength of electron-phonon coupling - is independent of the choice of functional.

Recent $G W$ calculations have shown that electronelectron correlation plays an important role in the effects of electron-phonon coupling on the band gap of diamond and GaAs [36], increasing the ZP correction within semilocal DFT by about $40 \%$. We have estimated the importance of electron-electron correlation by performing calculations with the HSE06 functional [17, 18], which has been shown to provide improved static lattice band gaps within DFT. We find somewhat smaller corrections than those reported for diamond and GaAs using $G W$ but, as in Ref. [36], these corrections increase the ZP renormalisation (details are provided in the Supplementary Material [51]).

The quadratic approximation of Eq. (2) is a computationally efficient approach for evaluating the ZP correction to the band gap. Furthermore, it provides access to the microscopic origins of the electron-phonon 


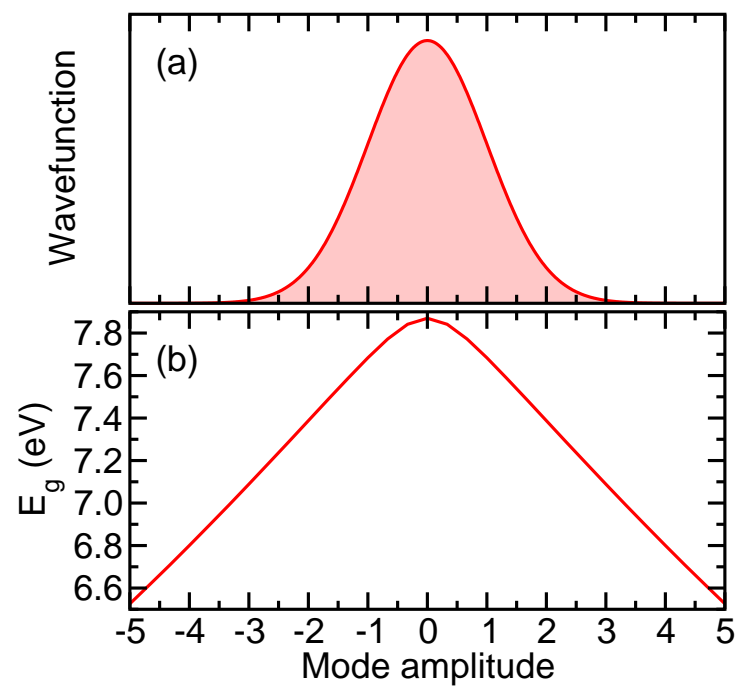

FIG. 2. (a) Wave function and (b) band gap $E_{\mathrm{g}}$ as a function of normal mode amplitude (in units of $1 / \sqrt{2 \omega}$ ) for a highenergy vibrational molecular mode of the HF crystal.

coupling strength, as each vibrational normal mode is treated separately. The importance of higher order terms in the expansion of Eq. (2) is less well-understood. These terms have been found to be crucial for the electronphonon coupling strength in helium under terapascal conditions [41], but to be unimportant for silicon at ambient pressure [43]. The question arises of how important they may be for the molecular crystals of interest here.

In Fig. 1 we compare the $\mathrm{ZP}$ correction to the band gaps of $\mathrm{CH}_{4}, \mathrm{NH}_{3}, \mathrm{H}_{2} \mathrm{O}$, and $\mathrm{HF}$, evaluated using the quadratic expansion and Monte Carlo sampling. The horizontal axes show the vibrational mode amplitude at which the band gap is calculated in order to evaluate the couplings $a_{n \mathbf{k}}^{(2)}$. The results reported in Fig. 1 correspond to primitive cells ( $\Gamma$-point sampling of the vibrational Brillouin zone), which are sufficient to illustrate the discrepancies between the two methods. Note that all other results in the paper are reported for larger simulation cells. We observe a strong dependence on the normal mode amplitude of the ZP correction evaluated within the quadratic approximation. It is clear that higher order terms in Eq. (2) are large, and should be included to obtain accurate results. We show an example of non-quadratic dependence in Fig. 2, corresponding to a normal mode describing a high-energy molecular vibration in HF. The dependence of $E_{\mathrm{g}}$ on mode amplitude has a near-linear behaviour at normal mode amplitudes larger than about $1 / \sqrt{2 \omega}$, and this non-quadratic region is clearly important given the width of the vibrational wave function shown.

Higher order terms in Eq. (2) have largely been neglected in the literature, and the results presented here and in Ref. 41 suggest that future studies should carefully assess their importance. Here we report accurate numeri- cal results from Monte Carlo evaluations and results from the quadratic expansion to investigate the microscopic origins of the electron-phonon coupling. Further comparisons of the quadratic and Monte Carlo approaches are given in the Supplementary Material [51].

In Fig. 3 we show schematic diagrams of the band gaps of the molecular crystals $\mathrm{CH}_{4}, \mathrm{NH}_{3}, \mathrm{H}_{2} \mathrm{O}$ and $\mathrm{HF}$. The dashed baseline corresponds to the static lattice band gap evaluated using the PBE-TS functional. The corrections arising from the screened-exchange HSE06 functional are indicated by the arrows labelled $\Delta E_{\mathrm{g}}^{\mathrm{HSE}}$, and the corrections induced by electron-phonon coupling at zero temperature are indicated by the coloured arrows labelled by $\Delta E_{\mathrm{g}}^{\mathrm{elph}}$. In each case the $\mathrm{ZP}$ correction to the band gap has been calculated with a large supercell using the PBE or PBE-TS functional. The Monte Carlo sampling approach has been used, achieving a statistical uncertainty smaller than $0.02 \mathrm{eV}$ in all cases. The ZP corrections obtained using the HSE06 functional are similar to those obtained with PBE-TS (see discussion in Supplementary Material [51]). It is worth mentioning that the static HSE06 gap for ice is significantly smaller than many-body perturbation theory estimates, which are in the range 9.2-10.1 eV [52, 53].

We observe very large vibrational $\mathrm{ZP}$ corrections to the band gaps across the four systems studied of above $1 \mathrm{eV}$. The corrections are comparable to those arising from the use of the HSE06 screened exchange functional when compared with the semi-local functionals. These giant $\mathrm{ZP}$ corrections are the largest found to date in any system at ambient conditions, and are about twice the ZP correction in diamond. We note that hydrogen and helium solids under extreme pressures have been reported to exhibit even larger ZP corrections [41, 42]. First-principles molecular dynamics calculations have also shown a large influence of thermal motion on the band gap of liquid water [54], of a size comparable to the electron-phonon effects reported in this work.

To investigate the microscopic origins of the strong electron-phonon coupling, we evaluate the contribution from individual normal vibration modes to the $\mathrm{ZP}$ correction using the quadratic method.

In $\mathrm{CH}_{4}$, the high frequency and virtually dispersionless molecular modes contribute about $69 \%$ of the overall ZP correction to the band gaps, and of these, the low energy twisting modes dominate, contributing as much as the combination of the higher energy stretching and wagging modes. The crystal modes contribute the remaining $31 \%$ to the overall $\mathrm{ZP}$ correction. In $\mathrm{NH}_{3}$, the molecular modes contribute about $55 \%$ of the overall $\mathrm{ZP}$ correction, with the low energy wagging modes dominating, followed by the high energy stretching modes, and finally the intermediate energy scissoring modes. The crystal modes contribute $45 \%$ to the overall ZP correction, and the dominant modes involve vibrations of hydrogen atoms which contribute $37 \%$ of the overall $\mathrm{ZP}$ 


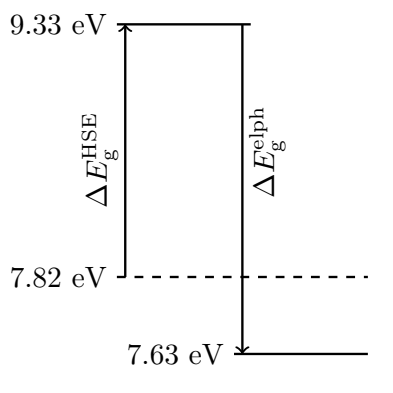

$\mathrm{CH}_{4}$

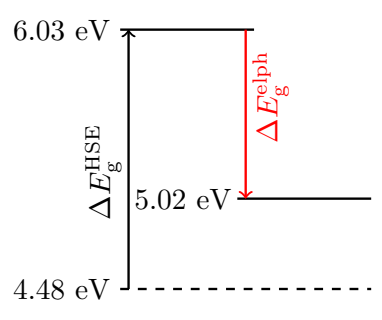

$\mathrm{NH}_{3}$

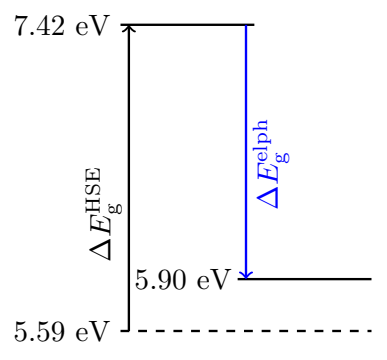

$\mathrm{H}_{2} \mathrm{O}$

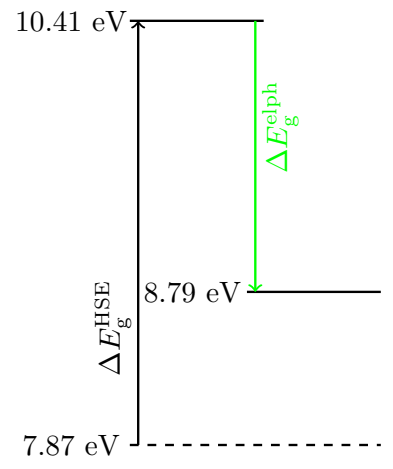

HF

FIG. 3. (color online) Band gap energies at the static lattice level with semi-local functionals (dashed baselines), the HSE06 correction $\Delta E_{\mathrm{g}}^{\mathrm{HSE}}$, and the $\mathrm{ZP}$ electron-phonon coupling correction $\Delta E_{\mathrm{g}}^{\mathrm{elph}}$.
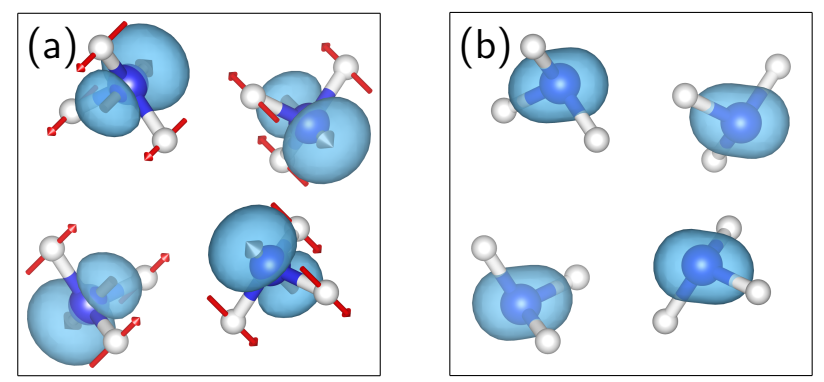

(c)

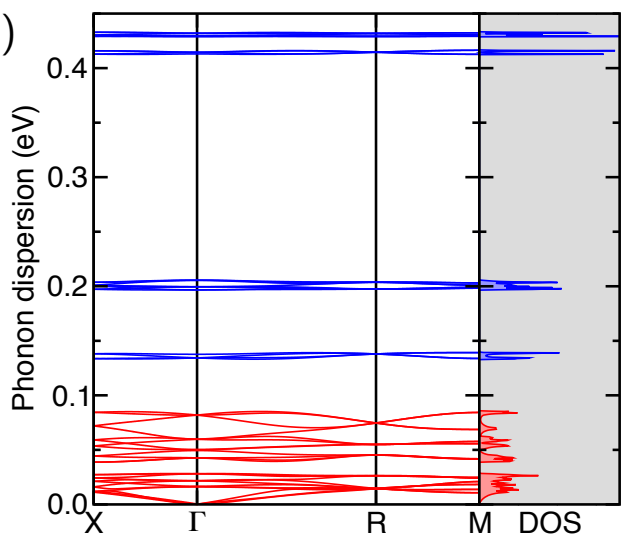

FIG. 4. (color online) Electronic charge density in crystalline $\mathrm{NH}_{3}$ corresponding to (a) the VBM and (b) the CBM. (c) Phonon dispersion (left) and DOS (right) of $\mathrm{NH}_{3}$, where the low-energy lattice modes are shown in red, and the highenergy molecular modes are shown in blue.

correction. For $\mathrm{H}_{2} \mathrm{O}$, the contributions from molecular and crystal modes are $46 \%$ and $54 \%$, respectively, and for $\mathrm{HF}$ they are $51 \%$ and $49 \%$.

The microscopic origin of the strength of electronphonon coupling can be understood by relating atomic distortions to changes in the charge density associated with the valence band maximum (VBM) and the conduction band minimum (CBM). As an example, we consider the $\mathrm{NH}_{3}$ molecular crystal. In Fig. 4 we show the charge density of the VBM and CBM for the $\mathrm{NH}_{3}$ molecular crystal. Both electronic states couple strongly to atomic vibrations. The phonon dispersion and density of states (DOS) of $\mathrm{NH}_{3}$ are also shown in Fig. 4, where the low-energy lattice modes are shown in red, and the molecular quasi-dispersionless high-energy modes are shown in blue. The VBM charge density distorts significantly under molecular vibrations, in particular the wagging modes in which the $\mathrm{H}$-atoms move in the direction of the charge density of the VBM (indicated as arrows in Fig. 4), explaining the strong coupling to it. These wagging modes exhibit some of the strongest electronphonon coupling in $\mathrm{NH}_{3}$. The charge density of the CBM is mostly affected by the low-energy lattice vibrational modes that involve collective $\mathrm{H}$-motion.

For comparison, we have also calculated the ZP contribution to the band gap of the isolated molecules. As observed for their crystalline counterparts, the molecular calculations also exhibit a strong non-quadratic behaviour, and we calculate Monte Carlo ZP corrections of $-0.39 \mathrm{eV}$ for $\mathrm{CH}_{4},-1.32 \mathrm{eV}$ for $\mathrm{NH}_{3},-0.48 \mathrm{eV}$ for $\mathrm{H}_{2} \mathrm{O}$, and $-0.04 \mathrm{eV}$ for HF. Apart from $\mathrm{NH}_{3}$, the molecular correction is significantly smaller in the molecules than in the crystals.

In conclusion, recent efforts to understand the effects of electron-phonon coupling in semiconductors have highlighted their importance for calculating accurate band structures using first-principles methods. We have reported the largest ZP corrections to band gaps found to date in solids under standard conditions of pressure, and we hope to motivate further studies of a wider range of systems. We have shown that the commonly used quadratic approximation fails for the description of the ZP correction to the band gap of hydrogen-rich molecular 
crystals. It would be interesting to assess the importance of dynamical effects on the calculated ZP corrections, but this is beyond the scope of the present work.

$G W$ calculations are often used to correct band gaps obtained with semi-local density functionals. $G W$ results are typically benchmarked against experimental data, but our results indicate that such comparisons could incur errors as large as $1 \mathrm{eV}$, and therefore care should be taken when assessing the accuracy of such calculations. The large ZP corrections found in molecular crystals could also be present in pharmaceutical drugs, polymers, or biological molecules.

Finally, we have highlighted the limits of the quadratic or AHC approximation for calculating band gap corrections arising from electron-phonon coupling. Most studies of these effects are based on the quadratic approximation, and our results call for a careful assessment of its validity over a wide range of materials.

B.M. acknowledges Robinson College, Cambridge, and the Cambridge Philosophical Society for a Henslow Research Fellowship. E.A.E. and R.J.N. acknowledge financial support from the Engineering and Physical Sciences Research Council (EPSRC) of the UK [EP/K013688/1]. The calculations were performed on the Cambridge High Performance Computing Service facility and the Archer facility of the UK's national high-performance computing service (for which access was obtained via the UKCP consortium [EP/K013564/1]).

*bm418@cam.ac.uk

[1] P. Hohenberg and W. Kohn, "Inhomogeneous electron gas," Phys. Rev. 136, B864-B871 (1964).

[2] W. Kohn and L. J. Sham, "Self-consistent equations including exchange and correlation effects," Phys. Rev. 140, A1133-A1138 (1965).

[3] M. C. Payne, M. P. Teter, D. C. Allan, T. A. Arias, and J. D. Joannopoulos, "Iterative minimization techniques for ab initio total-energy calculations: molecular dynamics and conjugate gradients," Rev. Mod. Phys. 64, 1045-1097 (1992).

[4] D. M. Ceperley and B. J. Alder, "Ground state of the electron gas by a stochastic method," Phys. Rev. Lett. 45, 566-569 (1980).

[5] J. P. Perdew and Alex Zunger, "Self-interaction correction to density-functional approximations for manyelectron systems," Phys. Rev. B 23, 5048-5079 (1981).

[6] John P. Perdew, Kieron Burke, and Matthias Ernzerhof, "Generalized gradient approximation made simple," Phys. Rev. Lett. 77, 3865-3868 (1996).

[7] R. W. Godby, M. Schlüter, and L. J. Sham, "Accurate exchange-correlation potential for silicon and its discontinuity on addition of an electron," Phys. Rev. Lett. 56, 2415-2418 (1986).

[8] R. W. Godby, M. Schlüter, and L. J. Sham, "Self-energy operators and exchange-correlation potentials in semiconductors," Phys. Rev. B 37, 10159-10175 (1988).
[9] W. M. C. Foulkes, L. Mitas, R. J. Needs, and G. Rajagopal, "Quantum Monte Carlo simulations of solids," Rev. Mod. Phys. 73, 33-83 (2001).

[10] Lubos Mitas and Richard M. Martin, "Quantum Monte Carlo of nitrogen: Atom, dimer, atomic, and molecular solids," Phys. Rev. Lett. 72, 2438-2441 (1994).

[11] A. J. Williamson, Randolph Q. Hood, R. J. Needs, and G. Rajagopal, "Diffusion quantum Monte Carlo calculations of the excited states of silicon," Phys. Rev. B 57, 12140-12144 (1998).

[12] Lars Hedin, "New method for calculating the one-particle Green's function with application to the electron-gas problem," Phys. Rev. 139, A796-A823 (1965).

[13] F Aryasetiawan and O Gunnarsson, "The GW method," Rep. Prog. Phys. 61, 237 (1998).

[14] Axel D. Becke, "A new mixing of Hartree-Fock and local density-functional theories," J. Chem. Phys. 98, 13721377 (1993).

[15] John P. Perdew, Matthias Ernzerhof, and Kieron Burke, "Rationale for mixing exact exchange with density functional approximations," J. Chem. Phys. 105, 9982-9985 (1996).

[16] J. Muscat, A. Wander, and N.M. Harrison, "On the prediction of band gaps from hybrid functional theory," Chem. Phys. Lett. 342, 397 - 401 (2001).

[17] J. Paier, M. Marsman, K. Hummer, G. Kresse, I. C. Gerber, and J. G. Ángyán, "Screened hybrid density functionals applied to solids," J. Chem. Phys. 124, 154709 (2006).

[18] J. Paier, M. Marsman, K. Hummer, G. Kresse, I. C. Gerber, and J. G. Ángyán, "Erratum: Screened hybrid density functionals applied to solids [J. Chem. Phys. 124, 154709 (2006)]," J. Chem. Phys. 125, 249901 (2006).

[19] Manuel Cardona and M. L. W. Thewalt, "Isotope effects on the optical spectra of semiconductors," Rev. Mod. Phys. 77, 1173-1224 (2005).

[20] H. Fesefeldt, "Der Einfluß der Temperatur auf die Absorptionsspektra der Alkalihalogenidkristalle," Z. Phys. 64, 623-628 (1930).

[21] C. D. Clark, P. J. Dean, and P. V. Harris, "Intrinsic edge absorption in diamond," Proc. R. Soc. London, Ser. A 277, 312-329 (1964).

[22] W. Bludau, A. Onton, and W. Heinke, "Temperature dependence of the band gap of silicon," J. Appl. Phys. 45, 1846-1848 (1974).

[23] S. Logothetidis, J. Petalas, H. M. Polatoglou, and D. Fuchs, "Origin and temperature dependence of the first direct gap of diamond," Phys. Rev. B 46, 4483-4494 (1992).

[24] P B Allen and V Heine, "Theory of the temperature dependence of electronic band structures," J. Phys. C 9, 2305 (1976).

[25] P. B. Allen and M. Cardona, "Theory of the temperature dependence of the direct gap of germanium," Phys. Rev. B 23, 1495-1505 (1981).

[26] Stefan Zollner, Manuel Cardona, and Sudha Gopalan, "Isotope and temperature shifts of direct and indirect band gaps in diamond-type semiconductors," Phys. Rev. B 45, 3376-3385 (1992).

[27] R. D. King-Smith, R. J. Needs, V. Heine, and M. J. Hodgson, "A first-principle calculation of the temperature dependence of the indirect band gap of silicon," Europhys. Lett. 10, 569 (1989). 
[28] Rodrigo B. Capaz, Catalin D. Spataru, Paul Tangney, Marvin L. Cohen, and Steven G. Louie, "Temperature dependence of the band gap of semiconducting carbon nanotubes," Phys. Rev. Lett. 94, 036801 (2005).

[29] Feliciano Giustino, Steven G. Louie, and Marvin L. Cohen, "Electron-phonon renormalization of the direct band gap of diamond," Phys. Rev. Lett. 105, 265501 (2010).

[30] Elena Cannuccia and Andrea Marini, "Effect of the quantum zero-point atomic motion on the optical and electronic properties of diamond and trans-polyacetylene," Phys. Rev. Lett. 107, 255501 (2011).

[31] E. Cannuccia and A. Marini, "Zero point motion effect on the electronic properties of diamond, trans-polyacetylene and polyethylene," Eur. Phys. J. B 85, 320 (2012).

[32] Bartomeu Monserrat, N. D. Drummond, and R. J. Needs, "Anharmonic vibrational properties in periodic systems: energy, electron-phonon coupling, and stress," Phys. Rev. B 87, 144302 (2013).

[33] Christopher E. Patrick and Feliciano Giustino, "Quantum nuclear dynamics in the photophysics of diamondoids," Nat. Commun. 4, 2006 (2013).

[34] Peng Han and Gabriel Bester, "Large nuclear zero-point motion effect in semiconductor nanoclusters," Phys. Rev. B 88, 165311 (2013).

[35] S. Poncé, G. Antonius, P. Boulanger, E. Cannuccia, A. Marini, M. Côté, and X. Gonze, "Verification of first-principles codes: Comparison of total energies, phonon frequencies, electron-phonon coupling and zeropoint motion correction to the gap between ABINIT and QE/Yambo," Comput. Mater. Sci. 83, 341 - 348 (2014).

[36] G. Antonius, S. Poncé, P. Boulanger, M. Côté, and X. Gonze, "Many-body effects on the zero-point renormalization of the band structure," Phys. Rev. Lett. 112, 215501 (2014).

[37] Bartomeu Monserrat and R. J. Needs, "Comparing electron-phonon coupling strength in diamond, silicon, and silicon carbide: First-principles study," Phys. Rev. B 89, 214304 (2014).

[38] Ion Garate, "Phonon-induced topological transitions and crossovers in Dirac materials," Phys. Rev. Lett. 110, 046402 (2013).

[39] Kush Saha and Ion Garate, "Phonon-induced topological insulation," Phys. Rev. B 89, 205103 (2014).

[40] A. J. Heeger, S. Kivelson, J. R. Schrieffer, and W. P. Su, "Solitons in conducting polymers," Rev. Mod. Phys. 60, 781-850 (1988).

[41] Bartomeu Monserrat, N. D. Drummond, Chris J. Pickard, and R. J. Needs, "Electron-phonon coupling and the metallization of solid helium at terapascal pressures," Phys. Rev. Lett. 112, 055504 (2014).
[42] Miguel A. Morales, Jeffrey M. McMahon, Carlo Pierleoni, and David M. Ceperley, "Towards a predictive first-principles description of solid molecular hydrogen with density functional theory," Phys. Rev. B 87, 184107 (2013).

[43] Christopher E Patrick and Feliciano Giustino, "Unified theory of electron-phonon renormalization and phononassisted optical absorption," J. Phys. Condens. Matter 26, 365503 (2014).

[44] X. Gonze, P. Boulanger, and M. Côté, "Theoretical approaches to the temperature and zero-point motion effects on the electronic band structure," Ann. Phys. 523, 168-178 (2011).

[45] P.B. Allen and J.C.K. Hui, "Thermodynamics of solids: Corrections from electron-phonon interactions," Z. Phys. B 37, 33-38 (1980).

[46] Stewart J. Clark, Matthew D. Segall, Chris J. Pickard, Phil J. Hasnip, Matt I. J. Probert, Keith Refson, and Mike C. Payne, "First principles methods using CASTEP," Z. Kristallogr. 220, 567 (2005).

[47] David Vanderbilt, "Soft self-consistent pseudopotentials in a generalized eigenvalue formalism," Phys. Rev. B 41, 7892-7895 (1990).

[48] Hendrik J. Monkhorst and James D. Pack, "Special points for Brillouin-zone integrations," Phys. Rev. B 13, 5188-5192 (1976).

[49] Alexandre Tkatchenko and Matthias Scheffler, "Accurate molecular van der Waals interactions from ground-state electron density and free-atom reference data," Phys. Rev. Lett. 102, 073005 (2009).

[50] Stefan Grimme, "Semiempirical GGA-type density functional constructed with a long-range dispersion correction," J. Comput. Chem. 27, 1787-1799 (2006).

[51] See Supplemental Material at [...] for details on the crystal structures, choice of exchange-correlation functional, supercell size convergence, and HSE ZP correction.

[52] P. H. Hahn, W. G. Schmidt, K. Seino, M. Preuss, F. Bechstedt, and J. Bernholc, "Optical absorption of water: Coulomb effects versus hydrogen bonding," Phys. Rev. Lett. 94, 037404 (2005).

[53] Changming Fang, Wun-Fan Li, Rik S. Koster, Jiri Klimes, Alfons van Blaaderen, and Marijn A. van Huis, "The accurate calculation of the band gap of liquid water by means of GW corrections applied to plane-wave density functional theory molecular dynamics simulations," Phys. Chem. Chem. Phys. 17, 365-375 (2015).

[54] Ding Pan, Quan Wan, and Giulia Galli, "The refractive index and electronic gap of water and ice increase with increasing pressure," Nat. Commun. 5, 3919 (2014). 